\title{
El perfil de la educación ambiental en América Latina y el Caribe: Un corte transversal en el marco del Decenio de la Educación para el Desarrollo Sustentable ${ }^{1}$
}

\author{
Edgar J. González-Gaudiano² \\ Julio C. Puente-Quintanilla ${ }^{3}$
}

Resumen: El artículo presenta los resultados de un estudio sobre la trayectoria actual de la educación ambiental realizado en nueve países de la región de América Latina y el Caribe, durante 2008 y 2009. La intención era evaluar los efectos regionales del plan de aplicación internacional del Decenio de la Educación para el Desarrollo Sustentable, a cinco años de haber entrado en operación, para caracterizar las tendencias y oportunidades regionales en el campo de la educación ambiental, y delinear estrategias apropiadas para su fortalecimiento y consolidación, a la luz de las tensiones que origina la aparición del campo de la educación para el desarrollo sustentable

Palabras clave: educación ambiental, desarrollo sustentable, DEDS.

Abstract: This article presents the findings of a study on the current trend in environmental education carried out in nine countries of the region of Latin America and the Caribbean during 2008 and 2009. The intention was to evaluate the regional effects of the plan to implement the Decade of Education for Sustainable Development internationally five years after having gone into operation. This will characterize regional trends and opportunities in this field of education and map out appropriate strategies for strengthening and consolidating them in light

${ }^{1}$ Financiamiento otorgado por Promep al proyecto clave 103.5/07/2523

2 Investigador Titular del Instituto de Investigaciones en Educación de la Universidad Veracruzana, México.

3 Profesor Investigador Titular del Instituto de Investigaciones Sociales de la Universidad Autónoma de Nuevo León, México. Gustavo Aguirre de la Cerda fungió como asistente de investigación durante los dos años de operación del proyecto. 
of the tension originating from the appearance of education for sustainable development.

Key words: environmental education, sustainable development, DESD.

\section{Introducción}

Intentar caracterizar la tensión existente entre la educación ambiental (EA) y la educación para el desarrollo sustentable (EDS) va más allá de una mera curiosidad epistémica. La EA y la EDS son dos proyectos político-pedagógicos distintos inmersos en una disputa por hegemonizar el campo de la educación ambiental, con sus implicaciones en cuanto a instalar una propuesta educativa estratégica que contribuye a definir de determinadas maneras la relación ser humano-medio ambiente. ${ }^{4}$

Por el lado de la educación ambiental, como práctica pedagógica-política, el proyecto tiende hacia un análisis crítico de la realidad socio-ambiental en el que prime su transformación en pro de un desarrollo humano responsable. Por el de la educación para el desarrollo sustentable, el proyecto es proclive a salvaguardar los valores y principios de un sistema económico que ha generado la obscena desigualdad mundial existente y una crisis ecológica sin precedente alguno en la historia del planeta. En pocas palabras, un sistema que produce pobres y páramos.

Por ello, en lo general, la contienda EA-EDS se halla enmarcada en el debate amplio de la globalización neoliberal y sus propósitos de imponer una sombría concepción del mundo que tiende a perpetuar un orden económico y político socialmente injusto, inequitativo y ambientalmente suicida, al llevar implícita una trayectoria de colisión civilizatoria.

No asumimos, sin embargo, que la educación ambiental sea un campo homogéneo y armónico, que sale al encuentro en todos los

${ }^{4}$ Para mayor información sobre el debate EA-EDS desde el punto de vista de diversos autores, véase González-Gaudiano (2008). Este texto incluye las opiniones de educadores ambientales de España, Canadá, Australia, Sud África, Nueva Zelanda y México sobre el este debate. 
casos de una praxis política en busca de formas democráticas socialmente justas para conservar la integridad de los ecosistemas. Como en todo espacio social, en la educación ambiental concurren diversos discursos con sus correspondientes trasfondos ideológicos y propuestas pedagógicas. Eso es válido también para la educación para el desarrollo sustentable, porque es justo admitir que no todas las propuestas de la educación para el desarrollo sustentable se encuentran alineadas al discurso del globalizado consumismo neoliberal. ${ }^{5}$

No obstante, la radicalización planteada arriba alude a las connotaciones más intrínsecas; esto es, a las huellas discursivas originarias y a sus principios fundacionales, tanto de la educación ambiental como de la educación para el desarrollo sustentable. No es lo mismo, por ejemplo, asumir como en la EA la naturaleza socialmente conflictiva de la crisis ambiental que, como hace la EDS, verla obsecuentemente como un simple desajuste de las fuerzas del mercado que puede resolverse mejorando la funcionalidad del sistema. Aún más, este desajuste pretende lograrse con base en un cándido optimismo sobre las potenciales aportaciones de la ciencia y sobre todo de la tecnología.

Nuestra tesis es que la EA ha permanecido como en un palimpsesto cuyo discurso pretendió ser borrado por la EDS sobreescribiendo un nuevo texto que distorsiona el mensaje original. ${ }^{6} \mathrm{No}$ hay duda alguna que la EDS ha querido proyectarse desde el marco institucional construido por la EA durante las últimas tres décadas, pero no así desde su plataforma teórica y política.

Por ello es que combatir el intento de desplazamiento impuesto desde arriba y afuera que se pretende hacer a través de la educación

${ }^{5}$ Véase por ejemplo Tilbury \& Wortman (2004).

${ }_{6}^{6}$ Palimpsesto proviene de la voz griega $\pi \alpha \lambda \square \mu \psi \eta \sigma \tau o \varsigma$ que significa borrado nuevamente y designa al manuscrito que aún conserva en su superficie huellas de una inscripción anterior, que fue eliminada deliberadamente para sobre-escribir un nuevo texto. Era una costumbre durante la edad media como una forma de reciclado por la escasez de pergamino. Muchos textos griegos clásicos ocultos por escritos cristianos que se consideraban perdidos han podido recuperarse, porque el borrado afortunadamente fue imperfecto. 
para el desarrollo sustentable, dado el alto costo para los países que se encuentran en diferentes fases del proceso de posicionamiento y consolidación del campo de la educación ambiental, implica conocer el tipo de proyectos que se están promoviendo en la región, sus rasgos y características tanto de los proyectos mismos como de los agentes que los impulsan.

A este respecto, hay que reconocer el significativo cambio de discurso que se ha operado en la UNESCO al respecto de dicho intento de desplazamiento. En el más reciente reporte preparado por Arjen Wals (UNESCO, 2009, p.72), se propone como una de las estrategias para la segunda mitad del Decenio, establecer sinergias con otras "educaciones adjetivadas", particularmente con la educación ambiental a fin de crear procesos que resulten en un mutuo enriquecimiento, de "resolver" las tensiones existentes y de contribuir a una mejorada conceptualización e institucionalización de ambas. Este cambio de discurso, que ya no afirma el agotamiento y extinción de la EA, se da precisamente por los precarios resultados obtenidos en posicionar apropiadamente a la EDS, pese a los esfuerzos emprendidos desde 1992. El mismo reporte (UNESCO, 2009, pp. 9 y 10) demuestra fehacientemente la fortaleza del campo de la EA. En una búsqueda a través de Google realizada entre 2005 y 2009, el tema de la EDS pasó de 89,000 a 215,000 websites (un crecimiento de 142\%), mientras la EA durante el mismo periodo tuvo un crecimiento de 133\% al pasar de 1.5 millones a 3.5 millones de websites. Más de dieciséis veces lo reportado para la EDS.

\section{El proyecto de investigación}

Por lo tanto y con el propósito de conocer lo que está sucediendo en América Latina y el Caribe a este respecto, entre 2008 y 2009 se llevó a cabo un estudio a fin de hacer esa identificación y estar en condiciones de desarrollar un análisis de tendencias y oportunidades regionales en el campo de la educación ambiental, y finalmente para delinear estrategias apropiadas para su fortaleci-miento y consolidación. El estudio se propuso entonces identificar las nuevas trayectorias de la educación ambiental en América Latina, a la luz de las tensiones que 
origina la aparición del campo de la educación para el desarrollo sustentable, específicamente:

a) Caracterizar la educación ambiental que se practica en la región, a partir de una muestra de proyectos y programas que se realizan en nueve países latinoamericanos, en términos de sus orientaciones conceptuales, la población a la que están dirigidos, sus agentes de promoción y la forma de inclusión de componentes sociales, políticos y económicos.

b) Identificar en dichos proyectos y programas las articulaciones existentes de la educación ambiental con los temas que, de acuerdo al plan de aplicación internacional del Decenio a cargo de la UNESCO, constituyen la educación para el desarrollo sustentable.

c) Definir las tendencias y escenarios más visibles para la educación ambiental en la región, de acuerdo con el ideario y la posición de representantes influyentes del campo.

La propuesta inicial consideraba la participación de sólo seis países para reunir alrededor de treinta proyectos. Sin embargo, las respuestas recibidas de los seis países inicialmente auscultados llegaron con lentitud y estuvieron por debajo de lo esperado. Además, otros países no considerados al enterarse del proyecto solicitaron participar, por lo que decidimos ampliar su número para obtener la cifra de proyectos requerida. La convocatoria se emitió por vía electrónica a los directorios y redes de educadores ambientales existentes en la región, invitando a los potenciales interesados a participar en el proyecto de investigación.

$\mathrm{Al}$ ir surgiendo los interesados se les informaba con más detalle de los objetivos y se les enviaba un primer formulario. La petición consistía en llenar un sencillo cuestionario de preguntas abiertas para recoger datos de corte cualitativo. Se solicitó que fueran los líderes de los proyectos quienes proporcionaran la información correspondiente. Como los proyectos participantes no se seleccionaron de una muestra aleatoria obtenida de un inventario regional de proyectos de educación ambiental —del cual carecemos_, ni su número permite hacer inferencias medianamente generalizables, tenemos claro que los 
resultados de este estudio no pueden considerarse representativos y mucho menos concluyentes. ${ }^{7}$

No obstante, sí le reconocemos a los resultados de este estudio un valor indicativo y exploratorio que no es desdeñable, sobre todo para plantear ulteriores estudios y tratar de obtener lo que Clifford Geertz (1973) denomina una descripción densa; es decir, recuperar la dimensión detallada y a profundidad de una realidad particular, interpretando lo observado para rescatar el discurso social en la jerarquía de sus estructuras de significación y poder construir una explicación inteligible. ${ }^{8}$

La información recabada se procesó en dos niveles. El primero mediante el ordenamiento de los datos recibidos que fueron organizados en categorías para desarrollar una base de datos cuantitativa. Las categorías no fueron pre-establecidas, sino que se construyeron con base en las respuestas proporcionadas que fueron agrupándose según sus características constituyendo pequeños conglomerados, cada uno de los cuales fue designado como categoría.

Un segundo nivel de procesamiento se realizó utilizando un software analítico llamado "Tropes" Versión en español 7.0, el cual permitió aplicar un análisis de contenido para identificar palabras clave con las cuales se generó un segundo cuestionario con variables de control para validar y complementar las respuestas obtenidas en la aplicación del primero.

7 Se insistió en varias ocasiones con los responsables de proyectos muy consolidados y creativos en varios países de la región que se animaran a participar, varios de los cuales habían publicado excelentes reportes en Tópicos en Educación Ambiental, pero no se obtuvo respuesta. Algunos de ellos que han escuchando la presentación de los resultados preliminares en foros regionales, ahora señalan que el perfil del campo que ha derivado del análisis de este estudio hubiera sido otro.

8 Un ejercicio de esta naturaleza implicaría un diseño metodológico cualitativamente distinto que necesariamente tendría que contemplar la observación in situ, así como la aplicación de técnicas e instrumentos que en esta ocasión no estuvieron dentro de las posibilidades económicas del estudio. 
El segundo cuestionario se elaboró con base en preguntas cerradas y de orden cuantitativo y se propuso verificar el contenido previamente manifestado por los participantes. Los cuestionarios fueron capturados y codificados en una base de datos para su proceso empleando el software de análisis estadístico para aplicación a las ciencias sociales "SPSS Versión 16.0". Se procesaron interrelaciones entre las variables de doble y hasta triple cruce, aunque al final consideramos que dada la falta de representati-vidad de la muestra de proyectos no se justificaba llegar a tal especificidad.

Las respuestas provinieron de instituciones y organizaciones en nueve países de la región de América Latina y el Caribe y se levantó un inventario de 31 proyectos y programas en marcha, tanto de educación formal como no formal. La información procesada consistía tanto de las respuestas a los dos cuestionarios como de la revisión de la documentación que, en algunos de los casos, se anexó a los mismos. ${ }^{9}$ Los proyectos se desarrollan en:

\begin{tabular}{|c|c|c|}
\hline País & Frecuencia & Porcentaje \\
\hline México & 10 & 32.26 \\
\hline Perú & 4 & 12.9 \\
\hline Brasil & 4 & 12.9 \\
\hline Colombia & 4 & 12.9 \\
\hline Cuba & 2 & 6.45 \\
\hline Venezuela & 2 & 6.45 \\
\hline Bolivia & 2 & 6.45 \\
\hline República Dominicana & 2 & 6.45 \\
\hline Argentina & 1 & 3.23 \\
\hline Total & $\mathbf{3 1}$ & $\mathbf{1 0 0 . 0 0}$ \\
\hline
\end{tabular}

9 A todos los casos seleccionados se les solicitó que anexarán información que pudiera brindar una mejor idea tanto del proyecto como de la organización, pero menos de la mitad de los participantes lo hicieron. 
Se realizaron también entrevistas breves sobre el tema a diez prominentes educadores latinoamericanos, las que han sido transcritas y procesadas y que operan como parte de una metodología de información cruzada para la contrastación de tendencias, mediante un análisis de discurso sobre fragmentos significativos de la información recabada para intentar hacer una lectura deconstructiva. Por razones de espacio, en este artículo se incluyen sólo algunos ejemplos de dicho material.

Un primer corte con los resultados obtenidos del análisis de los 31 proyectos se presentó en la conferencia central "Perspectivas regionales en educación ambiental: La contribución de Iberoamérica en el contexto de la ciudadanía planetaria", durante el VI Congreso Iberoamericano de Educación Ambiental, celebrado en la ciudad de San Clemente del Tuyu, Provincia de Buenos Aires, Argentina del 16 al 19 de septiembre de 2009; en la mesa redonda "Educación ambiental en América Latina" programada como parte de la agenda de trabajo del VI Congreso de Medio Ambiente de la Asociación de Universidades del Grupo de Montevideo, realizado en la Universidad Federal de São Carlos, en el estado de São Paulo, Brasil, del 5 al 8 de octubre de 2009, así como en el Encuentro Latinoamericano "A mitad del camino". Evaluación del Decenio de la Educación para el Desarrollo Sostenible 2005-2014, celebrado en Pátzcuaro, Michoacán, México del 12 al 14 de noviembre de 2009.

\section{Algunos resultados}

En la región, 35\% de los proyectos de educación ambiental siguen estando orientados principalmente hacia la conservación de los recursos naturales y la biodiversidad. 17\% tiene una orientación hacia el desarrollo regional y rural, y $11 \%$ hacia actividades relacionadas con la producción y consumo. Porcentajes menores se orientan hacia los demás temas que, según la UNESCO, caracterizan la educación para el desarrollo sustentable. Esto es preocupante en más de un sentido, y así lo registran varias de las entrevistas realizadas a los expertos, quienes consideran que aunque la educación ambiental en la región ha estado históricamente asociada con temas sociales, económicos y políticos, 
continúa anclada en un conjunto de prácticas atávicas. ${ }^{10}$ Podría pensarse que continúan prevaleciendo los enfoques conservacionistas y ecologistas que han sido promovidos por diversas organizaciones internacionales, quizá porque ha sido un buen mecanismo para allegarse recursos. Se verá este asunto del financiamiento más adelante.

“... existe una fuerte inercia de una educación todavía muy atrapada en el discurso de la conservación o de la solución por problemas como el de la basura o el del agua-, que está en algunos sentidos buscando ensanchar sus conceptos y sus prácticas, pero desafortunadamente no hay espacios de formación para poderlas consolidar y enriquecer; entonces, de manera optimista veo todavía prácticas atrofiadas por una inercia, aunque hay cada vez más educadores que están tratando de desenclaustrarse de las mismas a partir de un discurso crítico".

Javier Reyes, México

"Es verdad que hasta prácticamente los años noventa, la política ambiental se volcó sobre la conservación del medio ambiente, la información y la comunicación se centró en la conservación de la naturaleza —una naturaleza idealizada, la naturaleza salvaje, etc.- ; pero decir que la educación ambiental era conservacionista, es decir una media verdad, porque también se ha desarrollado desde el principio una educación ambiental más focalizada en el sistema de la madre naturaleza, en el consumo, muy crítica y muy futurista, en la línea de tratar de señalar la raíz de los problemas y no los fenómenos más periféricos del planeta. Por lo tanto esa acusación es cierta, pero no está contextualizada."

Pablo Meira, España

En cuanto al tipo de actividades que promueven los proyectos de educación no formal, sólo $17.7 \%$ de los mismos se orientan hacia la conservación del patrimonio natural y cultural, seguido de la organización comunitaria $15.6 \%$ y reciclaje/recuperación/ reducción/manejo

10 Véanse por ejemplo las etapas descritas por Solano (2009) y los comentarios de Tristão (2010) sobre las tendencias de la EA en el marco de la globalización. Para un reporte en materia de investigación en EA, véase González-Gaudiano y Lorenzetti (2009). 
de residuos 13.5\% y ahorro de agua y energía 11.5\%. Aquí llama la atención que las estrategias productivas alternativas es la actividad que recibió menos respuestas, lo que indica tal vez que nuestros proyectos están descuidando la adecuada vinculación con la problemática económica, incluso con el ecoturismo que suele ser una actividad muy socorrida en la región, toda vez que apenas $9.4 \%$ de los proyectos están relacionados con esta actividad. Esta aparentemente precaria capacidad de articulación de la educación ambiental no formal con alternativas productivas sería uno de los aspectos que merecería una exploración a profundidad, puesto que es fundamental en las estrategias regionales de conservación ecológica.

“... hay experiencias asombrosas de grupos, de organizaciones o de líderes con una visión orientadora - aunque no necesariamente tengan procedimientos o metodologías muy claras-, que elaboran propuestas mientras construyen esa relación entre sus ideas y la realidad. Son proyectos extraordinarios por la complejidad que manejan, por la forma como van innovando los métodos y lo ves tanto a nivel de las instituciones formales — de las escuelas - como a nivel de trabajo comunitario. Tienen mucho de esto mis colegas que trabajan desarrollo comunitario desde las ciencias sociales, como los antropólogos, aunque no asuman su trabajo como educación ambiental, pero son trabajos relacionados con el medio ambiente o con la relación comunidad y naturaleza. Son como chispazos de luz por su innovación, por su capacidad crítica y por la forma como asumen la complejidad, pero no son el enfoque dominante. La mayoría la constituyen proyectos inmediatistas, que buscan un efecto mediático, que buscan posicionarse ante alguna institución, ante algún sector o algún grupo político y hacerle el juego a alguna empresa a la que quieren conquistar para obtener recursos. Muchos son proyectos ingenuos, de gente muy lenta, sin formación para abordar la complejidad de las problemáticas ambientales y que cree que basta con la buena voluntad para hacer cosas. Es gente que no busca alternativas, sino que se queda con la información que le llega, son consumidores pasivos de discursos y de políticas".

Luz María Nieto Caraveo, México 
Por parte de los proyectos de educación formal, encontramos un mayor interés por vincular el currículum con la gestión escolar (20.5\%), así como con los problemas de la comunidad $(15.3 \%)$ y la ambientación curricular (10.4\%). Nuevamente llama la atención el interés -aunque no muy marcado- por encontrar mayores vínculos entre el currículum, la escuela y los problemas del entorno aledaño, elemento que ha sido promovido por las auditorías ambientales en España o los de certificación ambiental de escuelas que se puso en marcha en Chile, que lamentablemente no respondió a nuestra convocatoria.

Sin embargo, lo que surge con claridad de los resultados no es un interés institucional sino el de los actores locales. Como suele ocurrir con bastante frecuencia en nuestra región, pese a las grandes limitaciones y regulaciones existentes los actores locales rebasan a las instituciones responsables e impulsan por su cuenta iniciativas que les permiten gestionar el cambio de mejor manera y reoxigenar sus propios espacios laborales. Esto no ha sido fácil puesto que muchos de ellos se enfrentan a disposiciones institucionales que los constriñen a ciertos enfoques, políticas y programas diseñados desde instancias centrales.

"La idea era que los PRAES — los famosos Proyectos Ambientales Escolares_, con la ley de 1994, lograran actualizar todos los currícula; que la educación ambiental pasara de ser una cátedra a que todo estuviera ambientalizado. Por ejemplo, trabajar a partir del problema, a partir de la interdisciplina. Pero no ha sido así, desde hace unos ocho años el énfasis ha sido sostenibilista".

Patricia Noguera, Colombia

61\% de respuestas mencionan, sin embargo, que se vinculan con los problemas de la comunidad, promoviendo campañas (64\%), celebrando fechas especiales (61\%), induciendo la articulación del currículo y la gestión escolar (54\%) y la ambientación curricular (58\%), aunque los padres y la comunidad participan poco, apenas 35\%. 
Los sectores que no están en la ciudad pero habitan en zonas no urbanas o poco urbanizadas, tienen una vida cultural más referenciada en la naturaleza. Yo creo que ellos sí están reivindicando no sólo el derecho a la preservación de los recursos naturales y a la conservación de la naturaleza -e incluso los derechos de la naturaleza misma, en una posición extrema-, sino que están reivindicando derechos sociales concretos al acceso a los bienes naturales como camino para una calidad de vida y para obtener finalmente también los derechos sociales perdidos en este mismo movimiento de despojo.

En este sentido, sí creo que lo verde está relegado en la Argentina en este momento, como enfoque dentro de la educación ambiental. Porque estos sectores, que no casualmente son también sectores étnicos o grupos humanos que reivindican una visible articulación vital y productiva con los recursos naturales, reivindican un paquete de derechos que están -son- articulados. Este discurso, sin embargo, ocurre fuera de los ámbitos de la educación formal - de la educación institucionalizada, de la escuela-, porque es afuera donde está la vinculación directa con los movimientos sociales y ambientalistas que, a su vez, están articulados con las luchas sociales y entonces complejizan la demanda.

Dentro de la escuela, en cambio, paradójicamente, me parece que los componentes verde y tecnológico en el discurso de la educación ambiental todavía siguen siendo hegemónicos, lo cual habla muy mal del sistema educativo argentino que no pulsa la realidad del país, ni logra acercar su reflexión y los procesos educativos a la complejidad social inherente en la problemática ambiental. En el mejor de los casos el interés sigue centrado en la relación ambiente, población y desarrollo, una mirada funcionalista sobre el rol de la EA.

Pablo Sessano, Argentina

En el rubro de los obstáculos al proyecto, se confirman las sospechas de que la falta de financiamiento es el principal factor $(29 \%)$ aunque en un porcentaje menor de lo esperado; seguido de la carencia de apoyo de la autoridad (21\%) y la resistencia al cambio de actitudes y comportamientos $(19 \%)$. 


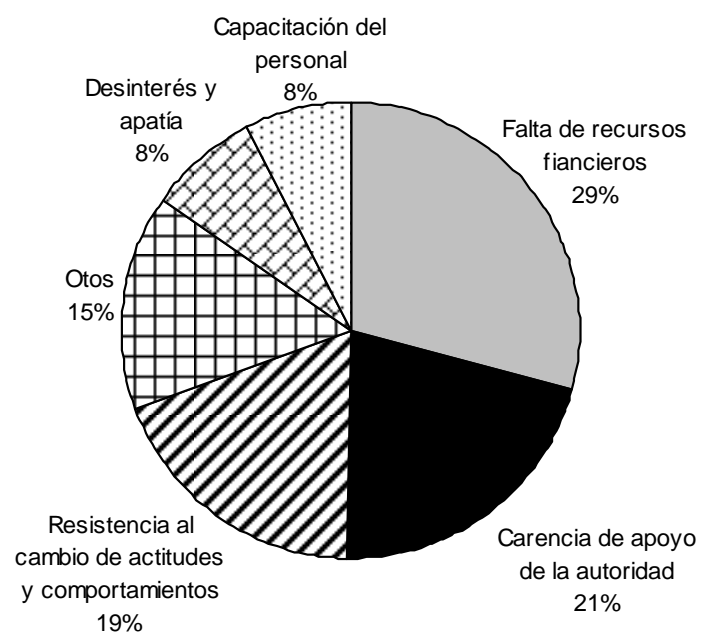

Estos dos problemas, el de la falta de apoyo de la autoridad (21\%), y el de las resistencias al cambio el que con el de desinterés y apatía, suma $27 \%$, son preocupantes porque indican que no estamos logrando crear las condiciones apropiadas para respaldar los proyectos en el mediano y largo plazos, ni estamos proporcionando los incentivos suficientes para motivar un mayor compromiso con nuestro tema. Sin embargo, somos bastante autocomplacientes ya que tan sólo un $8 \%$ de los obstáculos enfrentados los atribuimos a la capacitación del personal.

En cuanto al Decenio, podría decirse que encontramos una opinión optimista, puesto que la situación de la EA en la región no ha cambiado o incluso ha mejorado, en lo que respecta a los apoyos financieros, políticos, el interés de la comunidad por los temas ambientales y la comunicación entre quienes integramos la comunidad.

Ello ha provocado que la EA no esté siendo afectada o incluso esté fortaleciendo sus temas ambientales (55\%); aún así 33\% dice que se ha movido hacia temas sociales, económicos y políticos. 


\section{Apoyos Financieros}

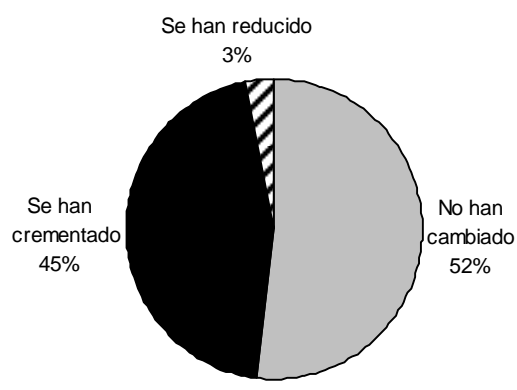

Interés de la comunidad por los temas ambientales

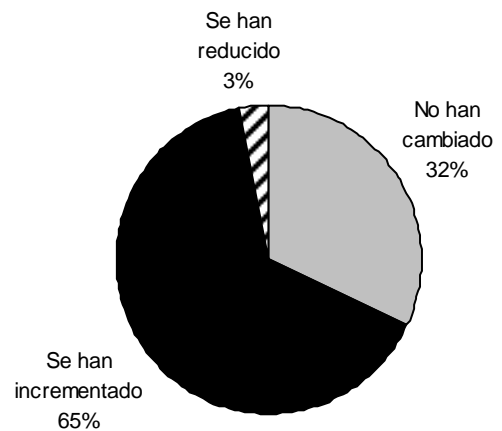

Apoyos Políticos e Institucionales

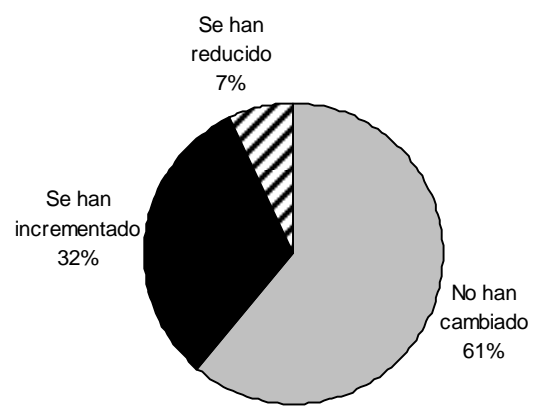

Comunicación entre los educadores ambientales

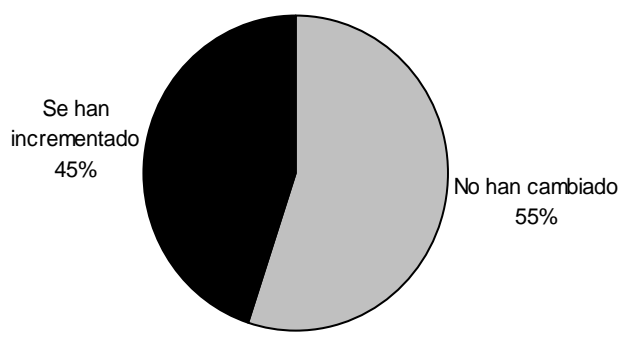

Haya disminuido sus

Sa haya movido hacia temas ambientales temas políticos $2 \%$

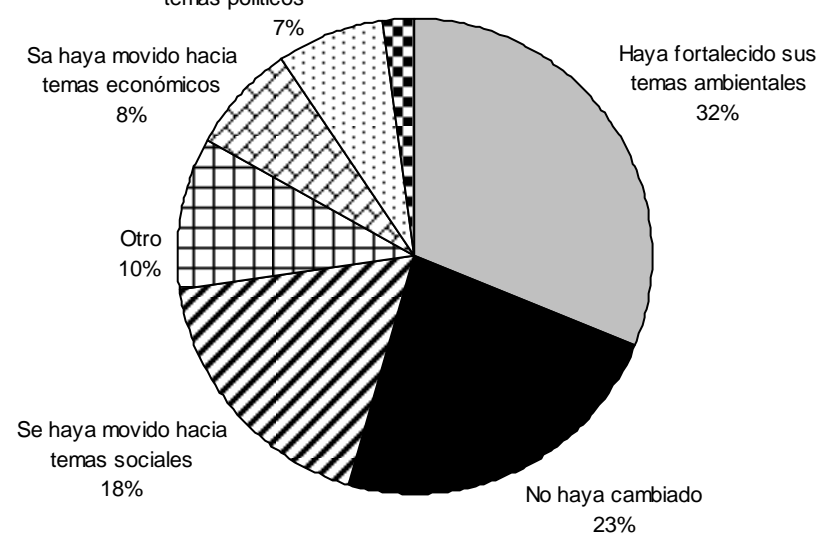


Frente a la pregunta específica de en qué ha contribuido la Década a su labor, 19\% dijo que en nada, 18\% no contestó, 7\% no abordó la pregunta y 36\% dijo que ha sido una influencia indirecta en concepciones políticas. El resto se distribuye en partes iguales en respuestas sobre mayor concientización y financiamiento, incremento del número de actividades y en la participación. Es decir, al parecer la Década ha ayudado un poco a posicionarnos mejor en el escenario de prioridades, pero no ha sido un factor relevante que haga una gran diferencia.

Existe mucha más información que puede ser analizada con un mayor detalle, por ejemplo a nivel de cada país, pero siendo el número de proyectos tan pequeño en algunos casos el análisis no tendría mucha validez. Véase sin embargo cómo responden los países a la pregunta sobre las contribuciones de la Década. ¿En qué ha contribuido la Década al Desarrollo de su labor ?

$\underline{\text { Cuba }}$

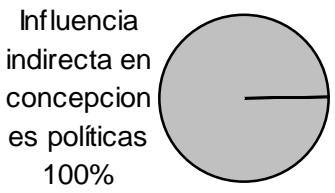

$\underline{\text { Bolívia }}$

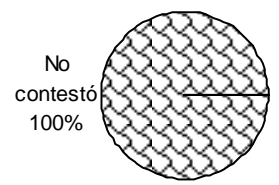

$\underline{\text { Argentina }}$

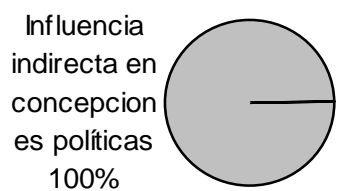

$\underline{\text { México }}$

$\underline{\text { Perú }}$ Ampliar el número de No respondió actividades la pregunta
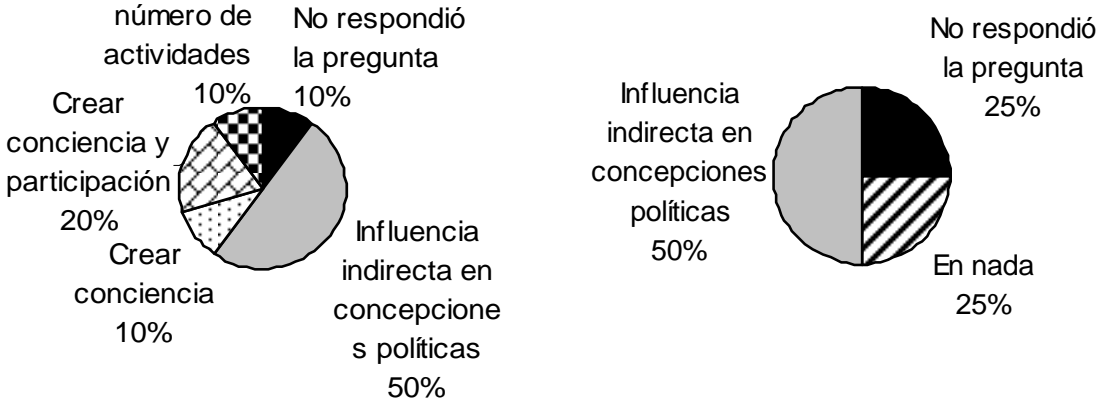


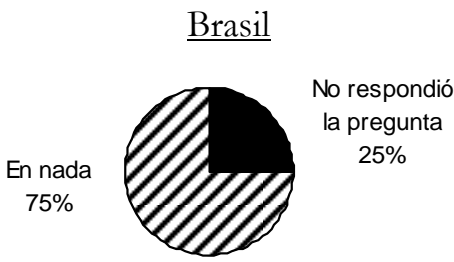

$\underline{\text { Colombia }}$

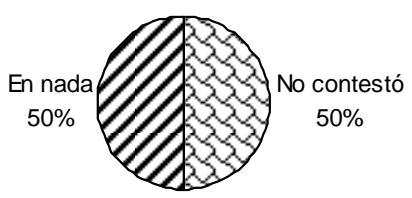

$\underline{\text { República Dominicana }}$

$\underline{\text { Venezuela }}$
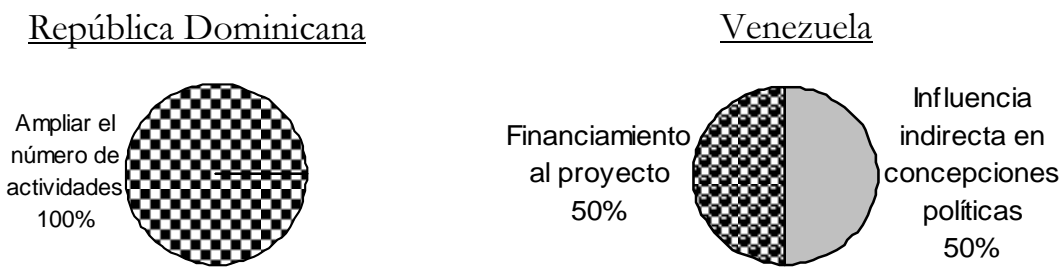

Pese a todo, consideramos que la información reportada por este ejercicio contribuye a tener un panorama que nos ayuda a entender algunos aspectos de la trayectoria que tiene la educación ambiental en la región, en el marco de la Década de la Educación para el Desarrollo Sustentable.

\section{Una conclusión abierta}

A lo largo de las tres últimas décadas hemos asistido a la construcción del campo de la educación ambiental en la región. Ha sido una construcción inestable y precaria tal y como lo es también la realidad de nuestros pueblos. Pero hemos sido testigos de un proceso bastante progresivo - no lineal- en el que la educación ambiental ha venido legitimándose en las políticas públicas y creando intersticios en el tejido social amplio. A esta construcción han contribuido significativamente los intercambios regionales entre educadores ambientales que cobraron fuerza a principios de la década de los años noventa y que han seguido consolidándose gracias ahora a las facilidades que ofrecen las nuevas tecnologías de la información y la comunicación. 
Sin embargo y pese a la circulación de todo el amplio conjunto de experiencias, debates y lecturas, observamos que prevalecen enfoques y aproximaciones que no logran trascender visiones muy limitadas frente al complejo problema ambiental. Estas visiones se traducen en propuestas que suelen responder puntualmente a los perfiles de proyectos solicitados por las fundaciones y organismos de financiación, ajustados a políticas ajenas a nuestras circunstancias, que desconocen las dificultades para poner en marcha acciones de educación ambiental en condiciones de severas carencias de satisfactores básicos o que se aferran a concepciones atávicas superadas.

Varios de los resultados de este proyecto de investigación han sido sorpresivos. Hace falta como dijimos ir a mayor profundidad, pero los datos están ahí y permiten inferir consecuencias que debemos atender en los espacios de nuestras reuniones, desarrollar otras estrategias y reinventar las formas de acercamiento a sectores y grupos clave. El propio Decenio de la EDS y la cobertura mediática que ha recibido el fenómeno del cambio climático crean condiciones que debemos aprovechar mejor en ese orden simbólico que nos constituye como sujetos de la educación ambiental.

\section{Referencias}

ARRUÉ, Rodrigo (Ed.). Balance y perspectivas de la educación ambiental en Chile e Iberoamérica. Tomo 1. Santiago de Chile, Comisión Nacional del Medio Ambiente.

GEERTZ, Clifford (1973). The interpretation of cultures. Selected essays. New York, Basic Books.

GONZÁLEZ-GAUDIANO, Edgar (Coord.) (2008). Educación, medio ambiente y sustentabilidad. Once lecturas críticas. México, Siglo XXI-UANL.

GONZÁLEZ-GAUDIANO, Edgar y Lorenzetti, L. (2009). "Investigação em Educação Ambiental na América Latina: mapeando tendências", em Educação em Revista. 25(3). pp. 191-211. 
GUERRA, Antonio F. S. e Figueiredo, M. L. (Orgs.) (2010). As sustentabilidades em diálogos. Itajaí, Editora da Univali.

SOLANO CORNEJO, David (2009). "Las cinco etapas de la educación ambiental” em Arrué (Ed.). pp. 73-79.

TILBURY, Daniela \& David Wortman (2004). Engaging people in sustainability. Commission on Education and Communication, IUCN, Gland, Switzerland and Cambridge, UK.

TRISTÃO, Martha (2010) “A educação ambiental e o paradigma da sustentabilidade em tempos de globalização", em Guerra, A. F. S. y Figueiredo, M. L. (Orgs.). pp. 157-170.

UNESCO (2009). Review of contexts and structures for Education for Sustainable Development 2009. Paris, UNESCO. Section for DESD Coordination.

\section{Proyectos participantes}

1. Programa de Formación Continua para la Educación Ambiental. Argentina.

2. Huertos Educativos Cochabamba. Bolivia

3. Diversificación Curricular para el Pantanal Boliviano. Bolivia.

4. Projeto Bairro Limpio. Brasil.

5. Programa de Formação em Educação Ambiental no Pantanal. Brasil.

6. Diagnóstico do Estado da Arte da Educação Ambiental no Espíritu Santo. Brasil.

7. Educación Socioambiental en la Perspectiva de la ONG EMCANTAR: Proyecto de educación formal y no formal en ciudades del interior de Brasil. Brasil.

8. Programa Jóvenes Gestores de Cuencas. Colombia.

9. Formación de Educadores y Líderes Comunitarios e Indígenas para la Conservación de la Biodiversidad Colombiana hacia un Desarrollo Sostenible. Colombia.

10. Foros Ambientales en el marco de la Década de la Educación para el Desarrollo Sostenible propuesta por la UNESCO. Colombia.

11. Libro de Educación Ambiental del Municipio de Falán y Conformación de los Grupos Ecológicos en las Instituciones y Centros Educativos de Falán Tolima- Colombia. "Falán Cuna de la Vida Silvestre". Colombia. 
12. Ahorro de Energía. Premisa para el Desarrollo Sostenible. Cuba.

13. Programa de Educación Ambiental para la Reorientación de la Cultura Local hacia el Desarrollo Sostenible de las Comunidades de la Ciénaga Occidental de Zapata. Cuba.

14. Campaña Educativa para Elevar la Conciencia de la Importancia Ambiental y Social del café de Sombra Mexicano. México.

15. Representaciones Sociales de las Relaciones con el Agua en el Mundo Urbano. México.

16. Programa de Educación Ambiental “Aprendiendo del Árbol”. México.

17. Trayectoria y Papel de los Investigadores en Educación Ambiental. México.

18. Programa de Educación Ambiental, Reserva de Biosfera Sierra de Huautla. México.

19. Diplomado Intervención Educativa Ambiental. México.

20. Consolidación de la Estrategia de Sensibilización y Educación Ambiental para la Eco Región Los Tuxtlas, Veracruz. México.

21. Juventud hacia un Golfo Norte Sustentable. Programa Integral Comunitario de Educación Ambiental. México.

22. Monitoreo Ecológico y Ambiental: Aves. México.

23. Educación Ambiental y Manejo de Ecosistemas en la Región de la Sierra de Manantlán, Jalisco. México.

24. Ciudad Limpia. Educación y Gestión Ambiental en Comunidades Educativas del Perú. Perú.

25. Introducción del Tema Ambiental en Forma Transversal en la Formación de Administradores. Perú.

26. Implementación del Sistema de Gestión Ambiental Escolar. Perú.

27. Ecoeducando. Perú.

28. Diplomado en Educación Ambiental. República Dominicana.

29. Adaptación Curricular a la Realidad Medioambiental de las Cuencas Altas de la Presa Sabana Yegua. República Dominicana.

30. Diseño e Implementación de un Programa de Educación Ambiental en Poblaciones Circunvecinas a la Laguna de Unare - Estado Anzoátegui. Venezuela.

31. Programa de Educación Ambiental "Fronti: el Oso Guaro va a tu Escuela". Venezuela.

\section{Artigo: recebido em 01/03/2010 - aprovado em 08/09/2010}

